\title{
LINC00461 affects the survival of patients with renal cell carcinoma by acting as a competing endogenous RNA for microRNA-942
}

\author{
YICHENG CHEN $^{1}$, JINKUI HE ${ }^{2}$, CHANGJU SU ${ }^{2}$, HUAN WANG ${ }^{1}$, YUANLEI CHEN ${ }^{1}$, \\ WENHAO GUO ${ }^{3}$, YINING $\mathrm{LI}^{3}$ and GUOQING DING ${ }^{1}$
}

\begin{abstract}
${ }^{1}$ Department of Urology, Sir Run-Run Shaw Hospital, College of Medicine, Zhejiang University, Hangzhou 310016;
${ }^{2}$ Department of Urology, Yiwu Traditional Chinese Medicine Hospital, Yiwu 322000;

${ }^{3}$ The Second Clinical Medical College of Fujian Medical University, Quanzhou 362000, P.R. China
\end{abstract}

Received December 3, 2018; Accepted July 26, 2019

DOI: 10.3892/or.2019.7311

\begin{abstract}
The present study aimed to investigate the potential mechanisms of human miR-942 in the sunitinib-resistance of renal cell carcinoma (RCC). A sunitinib-resistant OS-RC-2 cell line was established by continuous exposure to increasing concentrations of sunitinib for $\sim 12$ weeks. The expression levels of four miRNAs were determined by reverse transcription-quantitative (RT-q)PCR. miR-942 mimics were transfected into OS-RC-2 cells and RNA sequencing was performed on the miR-942- and negative control-transfected cells. Downregulated genes, including those of long non-coding RNAs (lncRNAs) and mRNAs, were identified. The target genes of miR-942 were predicted, followed by protein-protein interaction network construction and functional enrichment analyses of miR-942 target genes. In addition, RCC RNA-seq and miRNA-seq data were downloaded from The Cancer Genome Atlas (TCGA) database. The contributions of lncRNA and/or mRNAs to survival prediction were assessed and a competing endogenous RNA (ceRNA) network consisting of miR-942, lncRNA and mRNAs was constructed. The expression levels of LINC00461, miR-942, spalt-like transcription factor 1 (SALL1), methionyl aminopeptidase 1 (METAPl) and DDB1 and CUL4 associated factor $1(D C A F 11)$ were verified using RT-qPCR. The role of LINC00461 in cell viability was detected by MTT assay. The expression level of miR-942 was significantly increased in sunitinib-resistant cells. A total of seven lncRNAs and 155 mRNAs were predicted as target genes of miR-942 in the miR-942 mimic-treated samples, compared with the mimic control-treated group.
\end{abstract}

Correspondence to: Dr Guoqing Ding, Department of Urology, Sir Run-Run Shaw Hospital, College of Medicine, Zhejiang University, 3 East Qingchun Road, Hangzhou 310016, P.R. China E-mail: 3196014@zju.edu.cn

Key words: renal cell carcinoma, RNA sequencing, prognostic factors, competing endogenous RNA
These potential target genes were significantly associated with 'protein binding', 'TNF- $\beta$ signaling pathway', 'negative transcriptional regulation' and 'RNA binding'. Through the integrated analysis of RNA-sequencing and TCGA data, an miR-942-related ceRNA network, which was predicted to significantly affect the survival of patients with RCC, was constructed. The expression levels of lncRNA LINC00461 and the genes SALL1, METAP1, and DCAF11 were further verified. The viability of OS-RC-2 cells was decreased following co-transfection with miR-942 mimics and LINC00641 siRNA, and was comparable to that of wild type OS-RC-2 cells ( $\mathrm{P}>0.05)$. Therefore, IncRNA LINC00461 may act as an miR-942 ceRNA, and affect the survival of patients with RCC by regulating the expression of SALL1, METAP1 and DCAF11.

\section{Introduction}

Human renal cell carcinoma (RCC) accounts for $\sim 90 \%$ of all cancers of the kidney, in which significantly advanced, unresectable and metastatic RCC remains one of the most aggressive and fatal subtypes (1). Furthermore, the incidence of RCC is increasing at an annual rate of $\sim 1.5-5.9 \%$ worldwide (2). Generally, patients with RCC respond poorly to conventional radiotherapy and standard chemotherapy, which may be attributable to multidrug resistance (3). Molecular targeting agents such as immunotherapy, tyrosine kinase inhibitors and anti-angiogenic agents have become additional options for the treatment of metastatic RCC $(4,5)$. Sunitinib, a tyrosine kinase inhibitor of the vascular endothelial growth factor (VEGF) receptor, has been used as a first line treatment in patients with metastatic RCC. However, the initial treatment success of $\sim 80 \%$ is overshadowed by the occurrence of resistance after a drug-sensitive period (6). Therefore, it is critical to elucidate the mechanisms of RCC progression to identify novel biomarkers and develop advanced management options for patients with RCC.

MicroRNAs (miRNAs) are a class of endogenous short 18-25 nucleotide non-coding RNA molecules that bind to the 3'-untranslated region (UTR) of specific target mRNAs, thereby triggering mRNA degradation and translational 
repression (7). Increasing evidence has suggested that alterations in miRNA expression profiles contribute to cancer pathogenesis through various biological processes $(8,9)$. A recent study demonstrated that the antitumor miRNA (miR)-101-mediated ubiquitin-like with PHD and ring finger domains 1 pathway may be suppressed by sunitinib treatment (10). miR-21 has also been indicated to promote proliferation and differentiation, and decrease apoptosis in human RCC cells by activating the mTOR-STAT3 signaling pathway (11). Moreover, Machackova et al (12) showed that miR-429 inhibits the loss of E-cadherin in RCC cells, and is associated with poor prognosis in patients with RCC. Therefore, investigating the function of aberrantly expressed miRNAs and the mechanisms underlying miRNA regulation is important for elucidating the molecular mechanisms of RCC tumorigenesis, metastasis and drug resistance.

Prior et al (13) demonstrated that miR-942 was upregulated in sunitinib-resistant Caki-2 cells, compared with the sunitinib-sensitive counterparts. However, the exact role of miR-942 in the sunitinib response is far from clear. Furthermore, various long non-coding (lnc)RNAs have been proposed to function as competing endogenous RNAs (ceRNAs) that modulate miRNA target gene expression. In the present study, RNA sequencing was performed on miR-942-transfected and untransfected control cells. In addition, miRNA-seq and RNA-seq data were downloaded from The Cancer Genome Atlas (TCGA) (14), and comprehensive bioinformatics was used to analyze the significant functions involved. Integrated analysis of the RNA sequencing and TCGA data was performed to identify significant prognostic factors, and to construct an miR-942-related ceRNA network. The present study aimed to identify the target genes of miR-942 in promoting RCC cell proliferation and sunitinib resistance, which may aid in the development of novel therapies for RCC.

\section{Materials and methods}

Cell culture. Human renal cell carcinoma OS-RC-2 cells were purchased from the Shanghai Institute of Biological Sciences (Shanghai, China) and maintained in RPMI 1640 medium supplemented with $10 \%$ fetal bovine serum (Sigma-Aldrich; Merck $\mathrm{KGaA})$. The cells were incubated at $37^{\circ} \mathrm{C}\left(5 \% \mathrm{CO}_{2}\right)$ in a humidified incubator.

Establishment of sunitinib-resistant cell lines. The sunitinib-resistant OS-RC-2 cell line was established by continuous exposure to increasing concentrations of sunitinib (Selleck Chemicals) for $\sim 12$ weeks. The initial concentration of sunitinib was $1 \mu \mathrm{M}$, increased to $2 \mu \mathrm{M}$ after 4 weeks, to $5 \mu \mathrm{M}$ after 4 weeks, and maintained at $5 \mu \mathrm{M}$ for the last 4 weeks.

Quantification of miRNA in sunitinib-resistant cells. Reverse transcription-quantitative (RT-q)PCR was used to quantify four miRNAs in the sunitinib-sensitive and resistant cell lines. Total RNA was extracted from confluent cells in $60 \mathrm{~mm}$ culture dishes using TRIzol ${ }^{\circledR}$ reagent (Invitrogen; Thermo Fisher Scientific, Inc.), according to the manufacturer's protocol; $1 \mu \mathrm{g}$ total RNA was reverse-transcribed using HiScript Reverse Transcriptase (RNase H; Vazyme Biotech Co., Ltd.), and qPCR was performed using the SYBR ${ }^{\circledR}$-Green
Master Mix with the ABI 7500 system (Applied Biosystems). The thermocycling conditions were as follows: $50^{\circ} \mathrm{C}$ for $2 \mathrm{~min}$, $95^{\circ} \mathrm{C}$ for $10 \mathrm{~min}$, followed by 40 cycles of $95^{\circ} \mathrm{C}$ for $30 \mathrm{sec}$, and $60^{\circ} \mathrm{C}$ for $30 \mathrm{sec}$. All reactions were performed in triplicate and the expression values were normalized to that of U6 (15). The PCR primers are displayed in Table I.

Overexpression of $\mathrm{miR}-942$ in $O S-R C-2$ cells. The miR-942 mimic and mimic control were designed and synthesized by Shanghai GenePharma Co., Ltd. The sequences are as follows: miR-942 forward, 5'-UCUUCUCUGUUUUGGCCAUGUG-3', and reverse, 5'-CAUGGCCAAAACAGAGAAGAUU-3'; negative control forward, 5'-UUCUCCGAACGUGUCACGUTT-3', and reverse, 5'-ACGUGACACGUUCGGAGAATT-3'. Cells at $70-80 \%$ confluence were transfected with the miR-942 mimic or mimic control (100 nmol/l) using Lipofectamine 2000 transfection reagent (Invitrogen; Thermo Fisher Scientific, Inc.) following the manufacturer's protocol. Each group contained three replicates, and the cells were harvested $48 \mathrm{~h}$ post-transfection.

\section{RNA sequencing}

RNA isolation and sequencing. Total RNA was extracted from confluent cells in $60 \mathrm{~mm}$ culture dishes using TRIzol ${ }^{\circledR}$ reagent (Invitrogen; Thermo Fisher Scientific, Inc.), according to the manufacturer's protocol. The purity, concentration and quality of the RNA was assessed prior to sequencing using a NanoDrop Spectrophotometer (Thermo Fisher Scientific, Inc.), the Qubit 2.0 Fluorometer (Invitrogen; Thermo Fisher Scientific, Inc.) and the Agilent 2100 Bioanalyzer (Agilent Technologies, Inc.), respectively. Sequencing libraries were generated using the NEBNext ${ }^{\circledR}$ Ultra $^{\mathrm{TM}}$ RNA Library Prep kit for Illumina (cat. no. NEB \#E7530; New England Biolabs Inc.). The Qubit 2.0 Fluorometer and Agilent 2100 Bioanalyzer were then used to verify the quality, concentration and size of the cDNA libraries. The libraries were pooled and sequenced on an Illumina HiSeq platform, and raw reads were generated. The original sequencing data were uploaded to the NCBI SRA database (https://www.ncbi.nlm.nih.gov/sra) with the accession number SRP127372. Reads with adaptor sequences, those containing $>10 \%$ unknown nucleotide content, and those with low quality bases accounting for $>50 \%$ of the total nucleotides were filtered out during data processing.

Sequence alignment and identification of differentially expressed genes (DEGs). Filtered reads were aligned to the reference genome using Hierarchical Indexing For Spliced Alignment Of Transcripts (16). Initially, the data were normalized by counts per million (CPM), and low-level expressed genes (expression values $<0.1$ ) were filtered out. The quasi-likelihood F-tests method in the R package edge (17) was used to determine DEGs between the miR-942 mimicand the mimic control-treated group. A false discovery rate (FDR)-correction (18) was applied to account for multiple testing and false-positives, where the threshold was set at FDR $<0.05$. Furthermore, DEGs were classified as mRNAs and lncRNAs using genomic annotation information in the GENCODE (v. 24) database (19).

Prediction and screening of miR-942 target genes. Downregulated lncRNA and mRNA genes identified during 
Table I. Primers for microRNA reverse transcription-quantitative PCR in sunitinib-resistant OS-RC-2 cells.

\begin{tabular}{lll}
\hline Name & Orientation & Sequence (5'-3') \\
\hline U6 & Forward & CGCTTCGGCAGCACATATAC \\
hsa-miR-942-5p & Reverse & AAATATGGAACGCTTCACGA \\
& Reverse & CCAGTGCAGGGTCCGAGGTATT \\
hsa-miR-133a-5p & Forward & TGCGCTCTTCTCTGTTTTGGCC \\
& Reverse & CCAGTGCAGGGTCCGAGGTATT \\
hsa-miR-628-5p & Forward & TGCGCAGCTGGTAAAATGGAAC \\
& Reverse & CCAGTGCAGGGTCCGAGGTATT \\
hsa-miR-484 & Forward & TGCGCATGCTGACATATTTACT \\
& Reverse & CCAGTGCAGGGTCCGAGGTATT \\
& Forward & TGCGCTCAGGCTCAGTCCCCTC
\end{tabular}

Has-miR, Homo sapiens micro RNA.

differential expression analysis were considered as potential target genes for miR-942. miRanda software (v3.3a) (20) was used to predict the binding sites of miR-942 and these DEGs. The parameters were set as follows: -sc 120, -en 0, -strict. If the genes possessed a predicted miR-942 binding site, they were considered to be target genes.

Protein-protein interaction (PPI) analysis of predicted $m R N A$ s. For the resulting miR-942 target mRNAs, PPI analysis was performed using the Search Tool for the Retrieval of Interacting Genes/Proteins (21). In the present study, required confidence (combined score) $>0.4$ was selected as the screening threshold. Next, Cytoscape (22) was used to construct a PPI network and the topology of the network was analyzed. According to the ranking of network connectivity, important nodes in the PPI network were subsequently obtained.

Functional enrichment analysis of putative miR-942 target $m R N A s$. For the resulting putative miR-942 target mRNAs, Gene Ontology (GO) and Kyoto Encyclopedia of Genes and Genomes (KEGG) pathway enrichment analysis were performed on the DEGs using the Database for Annotation, Visualization and Integrated Discovery (v. 6.8) online tool (23). The parameters were set as gene count $\geq 2$, with a hypergeometric test significance threshold of $\mathrm{P}<0.05$. The results of enriched KEGG pathways and GO terms in the biological process, molecular function and cellular component categories (24) were obtained.

\section{Analysis of TCGA data}

Processing of TCGA data. Level 3 RNA-seq data containing the exon and clinical data of 606 samples for kidney renal clear cell carcinoma (KIRC) were downloaded from TCGA database (14), and the raw counts expression matrix was extracted. The data were re-annotated by integrating the genomic annotation information in the GENCODE database (V24lift) (19), along with the chromosomal location information of the exon. According to the re-annotation data, the genes were classified into lncRNAs and mRNAs. The expression values of multiple exons that corresponded to the same gene were integrated to obtain corresponding gene expression profiles. The gene expression matrix was subsequently normalized using the CPM method.

Level 3 miRNA-seq data from KIRC 588 samples were downloaded from the TCGA database (14). The data were also normalized by the CPM method, and the miR-942 expression values were extracted.

Gene set enrichment analysis (GSEA) of miR-942 and RNA-seq data from TCGA. For miRNA-seq and RNA-seq data, normal tissue samples and samples with only one type of data (e.g. miRNA-seq or RNA-seq) were excluded, leaving a total of 515 tumor tissue samples. Subsequently, the GSEA tool (25) was used to analyze the KEGG pathways influenced by miR-942. The coding gene expression profiles were set as the expression dataset and the miR-942 expression value as phenotype labels; GSEA analysis was performed using c2.cp.kegg.v6.1.symbols, primarily focusing on the enriched phenotype that was negatively correlated with miR-942.

Integrated analysis of RNA-sequencing and TCGA data Identification of prognostic factors. To evaluate the prognostic capacity of each gene, 533 patient samples from TCGA database that had clinical survival information and contained normalized target gene expression profiles were included. The analysis was based on the median gene expression values within these patient samples. The patients were then divided into high- and low-expression groups using the median gene expression values as the cutoff point. The difference in the survival rates of patients between the high- and low-expression groups was compared, and the genes potentially influencing the prognosis of patients were predicted. The $\mathrm{R}$ survival package (26) was used to construct Kaplan-Meier curves, and the survival curves were compared using the log-rank test. $\mathrm{P}<0.05$ was considered to indicate a statistically significant difference, and was used to assess the contribution of lncRNA and/or coding genes to survival prediction.

CeRNA network construction. The correlation coefficients for miRNA-lncRNA and miRNA-mRNAs identified from TCGA data portal were calculated using Pearson's correlation coefficient (27). The miRNA-IncRNA and miRNA-coding 
Table II. Primers for mRNA reverse transcription-quantitative PCR in OS-RC-2 cells.

\begin{tabular}{lll}
\hline Gene & \multicolumn{1}{c}{ Orientation } & \multicolumn{1}{c}{ Sequence (5'-3') } \\
\hline GAPDH & & TGACAACTTTGGTATCGTGGAAGG \\
& Reverse & AGGCAGGGATGATGTTCTGGAGAG \\
LINC00461 & Forward & CAGCCTATGACAGACAGCCC \\
& Reverse & CCAGTTGGTGCTGCCATTTG \\
METAP1 & Forward & CATCCAGGGCTCGTACTTCTG \\
& Reverse & TCTCGCTTCGCCTTTTCATCT \\
DCAF11 & Forward & AGCTTGGGATGGTCGTCTTG \\
& Reverse & TCTCTGGTGCAACATTCGAGG \\
SALL1 & Forward & GACGTGATGAACCAGATATTGCT \\
& Reverse & TTGACGAAAACGGCTTGTTAAAG \\
hsa-miR-942-5p & Reverse transcription & GTCGTATCCAGTGCAGGGTCCGAGGTATTCGCACTGGATACGACCACATG \\
& Stem-Loop & GCGCTCTTCTCTGTTTTGGC \\
\hline
\end{tabular}

METAP1, methionyl aminopeptidase 1; DCAF11, DDB1 and CUL4 associated factor 1; SALL1, spalt-like transcription factor 1; miR, micro RNA.

gene pairs with correlation coefficients $<0$ (i.e., negative correlation) and $\mathrm{P}<0.05$ indicated that there was a negative correlation between the expression of miRNA and lncRNA or coding gene. Moreover, these results were compared with the miR-942 target genes from the analysis of RNA-sequencing data. Overlapping target genes were identified as the final potential miR-942 target genes. The miR-942-related ceRNA network was constructed using Cytoscape software (22).

$R T-q P C R$. Total RNA from OS-RC-2 cells in confluent $60-\mathrm{mm}$ cell culture dishes was extracted using TRIzol ${ }^{\circledR}$ reagent (Invitrogen; Thermo Fisher Scientific, Inc.), according to the manufacturer's protocol; $1 \mu \mathrm{g}$ total RNA was reverse transcribed using the PrimeScript ${ }^{\mathrm{TM}}$ RT Master Mix (Takara Bio, Inc.). Subsequently, qPCR was performed using SYBR ${ }^{\circledR}$ Premix EX taq with the ABI 7500 system (Applied Biosystems; Thermo Fisher Scientific, Inc., with GAPDH as the reference gene, according to the manufacturer's protocol. The primers are listed in Table II. The thermocycling conditions were as follows: $50^{\circ} \mathrm{C}$ for $3 \mathrm{~min}, 95^{\circ} \mathrm{C}$ for $3 \mathrm{~min}$ and then 40 cycles of $95^{\circ} \mathrm{C}$ for $10 \mathrm{sec}$, and $60^{\circ} \mathrm{C}$ for $30 \mathrm{sec}$. The relative gene expression levels were calculated using the $2^{-\Delta \Delta \mathrm{Cq}}$ method (15).

lncRNA transfection. Small interfering (si)RNA LINC00641 and the negative control (NC) siRNA were purchased from Guangzhou RiboBio Co., Ltd., and $200 \mathrm{nmol} / \mathrm{l}$ of each was transfected into cells using Lipofectamine ${ }^{\circledR} 3000$ (Invitrogen; Thermo Fisher Scientific, Inc.,) according to the manufacturer's instructions. The mixture containing the synthesized nucleotide and the transfection reagent was incubated at room temperature for $10 \mathrm{~min}$ and subsequently added to the cultured cells. The cells were harvested $48 \mathrm{~h}$ post-transfection.

MTT assay. Cell viability was assessed using an MTT assay. Briefly, $100 \mu \mathrm{l}$ transfected cells were seeded into 96-well plates at a density of $\sim 5 \times 10^{3}$ cells/well. After $24 \mathrm{~h}, 0,2.5,5,10$, 15 or $20 \mu \mathrm{M}$ sunitinib was added and the cells were incubated for $48 \mathrm{~h}$. MTT $(10 \mu \mathrm{l}, 5 \mathrm{mg} / \mathrm{ml})$ was added to each well prior to culturing for a further $4 \mathrm{~h}$ at $37^{\circ} \mathrm{C}$. To dissolve the formazan crystals, $150 \mu \mathrm{l}$ DMSO was added to each well and shaken gently for $5 \mathrm{~min}$ at room temperature. The absorbance was then measured at $550 \mathrm{~nm}$ using a microplate reader.

Statistical analysis. Statistical analyses were performed using SPSS v13.0 (SPSS, Inc.) software. The independent samples t-test and Pearson's correlation analysis were used to compare the bioinformatics data. All values are expressed as the mean \pm standard deviation. The experimental results were analyzed using the unpaired, two-tailed Student's t-test (two groups) or ANOVA ( $\geq 3$ groups) followed by Bonferroni's correction. $\mathrm{P}<0.05$ was considered to indicate a statistically significant difference.

\section{Results}

Expression levels of miRNAs in sunitinib-resistant cells. A total of four miRNAs (miR-942-5p, miR-133a-5p, miR-484 and miR-628-5p) were quantified in sunitinib-sensitive or -resistant OS-RC-2 cells using RT-qPCR. As shown in Fig. 1A, the expression levels of miR-942-5p and miR-484 were significantly increased, whilst those of miR-133a-5p and miR-628-5p were significantly decreased in sunitinib-resistant cells, compared with those in sunitinib-sensitive cells $(\mathrm{P}<0.01)$. This result suggests that miR-942-5p and miR-484 may be associated with sunitinib resistance. As the fold-change of miR-942-5p was the largest between the sunitinib-resistant and sensitive cell lines, this miRNA was selected for further investigation.

Identification of differentially expressed lncRNAs and mRNAs using RNA-sequencing data. miR-942 mimics were successfully transfected into OS-RC-2 cells (Fig. 1B), and an MTT assay was used to assess cell viability at different concentrations of sunitinib (Fig. 1C). The MTT assay demonstrated that cell viability was modestly, but significantly increased following miR-942 transfection and treatment with 1 and $10 \mu \mathrm{M}$ sunitinib $(\mathrm{P}<0.01$ and $\mathrm{P}<0.05$, respectively; Fig. 1D), 

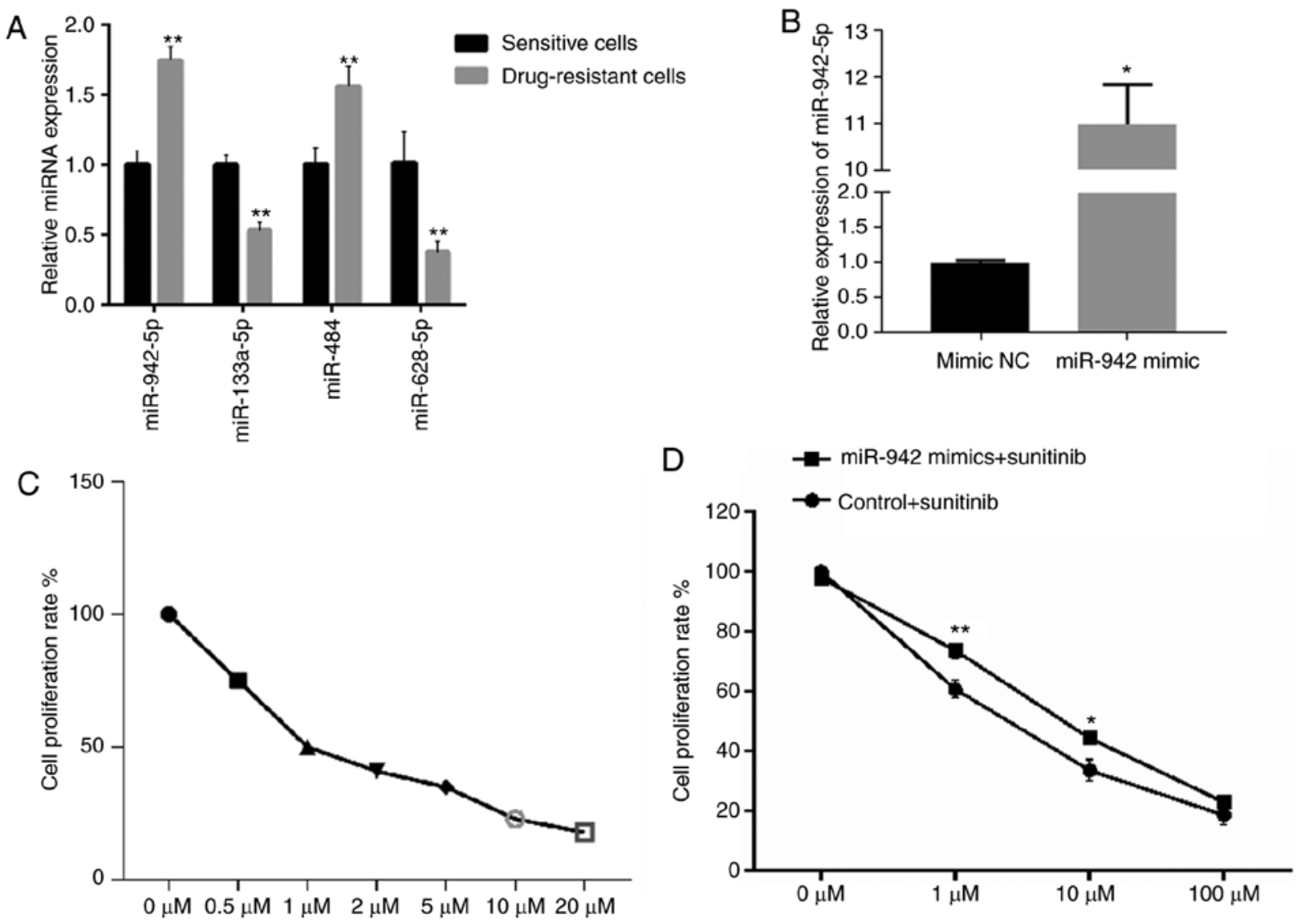

Figure 1. (A) Relative expression levels of miRNAs in sunitinib-sensitive and resistant OS-RC-2 cells. (B) Relative expression level of miR-942-5p in miR-942-5p-transfected and NC-transfected cells. (C) Proliferation rates of OS-RC-2 cells following sunitinib treatment. (D) Viability of miR-942-5pand NC-transfected OS-RC-2 cells following sunitinib treatment. ${ }^{*} \mathrm{P}<0.05$ and ${ }^{* *} \mathrm{P}<0.01$, sunitinib-sensitive and miR942-mimics transfected+sunitinib cells vs. sunitinib-sensitive and NC-transfected+sunitinib cells. miR, microRNA; NC, negative control.

suggesting that miR-942 may serve an important role in $\mathrm{RCC}$ cell proliferation and sunitinib resistance. RNA sequencing was conduced on the miR-942- and miR-942 NC transfected cells. With the threshold of FDR $<0.05$, a total of 95 differentially expressed lncRNAs, including 65 upregulated and 30 downregulated IncRNAs, were identified in the miR-942 mimic-treated group compared with the control-treated group. Concurrently, 1,697 differentially expressed mRNAs were screened out between the miR-942 mimic and the control-treated groups, comprising 1,171 upregulated and 526 downregulated differentially expressed mRNAs.

Prediction of miR-942 target genes. By combining the identified downregulated genes and the binding sites between miR-942 and the predicted lncRNA/mRNAs, seven lncRNAs and 155 mRNAs were predicted to be target genes of miR-942, respectively (Fig. 2A). Heat maps display the downregulation and upregulation of the top 10 DEGs (Fig. 2B) and the total DEGs (Fig. 2C).

Establishment of a PPI network based on miR-942 target DEGs. PPI network analysis was performed to evaluate which of the miR-942 targets served a vital role in patient prognosis. The PPI network contained 82 nodes and 148 edges (interactions), and the hub nodes with a higher connectivity degree included the heat shock protein family A (Hsp70) member 8 (HSPA8, degree=12), Dicer 1, ribonuclease III (DICER1, degree=12), dihydropyrimidine dehydrogenase (DPYD, degree=11), integrin subunit $\beta 5$ (ITGB5, degree=9), RAD23 Homolog $B$, nucleotide excision repair protein (RAD23B, degree $=8$ ), enhancer Of Zeste 2 polycomb repressive complex 2 subunit $(\mathrm{EZH} 2$, degree $=8)$, heat shock protein family D (Hsp60) member 1 (HSPD1, degree=8), DExD-Box helicase 21 (DDX21, degree=8), polypyrimidine tract binding protein 1 (PTBP1, degree=7), dyskerin pseudouridine synthase 1 ( $\mathrm{DKC} 1$, degree $=7)$, protein arginine methyltransferase 1 (PRMT1, degree $=7)$ and transketolase (TKT, degree=7) (Fig. 3).

GO and KEGG pathway enrichment analysis of miR-942 target DEGs. To further explore the function of miR-942 target DEGs, GO and KEGG pathway analysis was performed (Fig. 4). The identified target genes were found to be significantly associated with 'protein binding', 'TGF- $\beta$ signaling pathway', 'negative transcriptional regulation' and 'RNA binding'.

GSEA analysis of miR-942 and RNA-seq data from TCGA. After preprocessing of the RNA-seq and miR-942 data from TCGA, enrichment analysis was performed using the GSEA tool. The results of negative regulation with miR-942 were selected using a nominal $\mathrm{P}$-value of $<0.1$ as a significant 

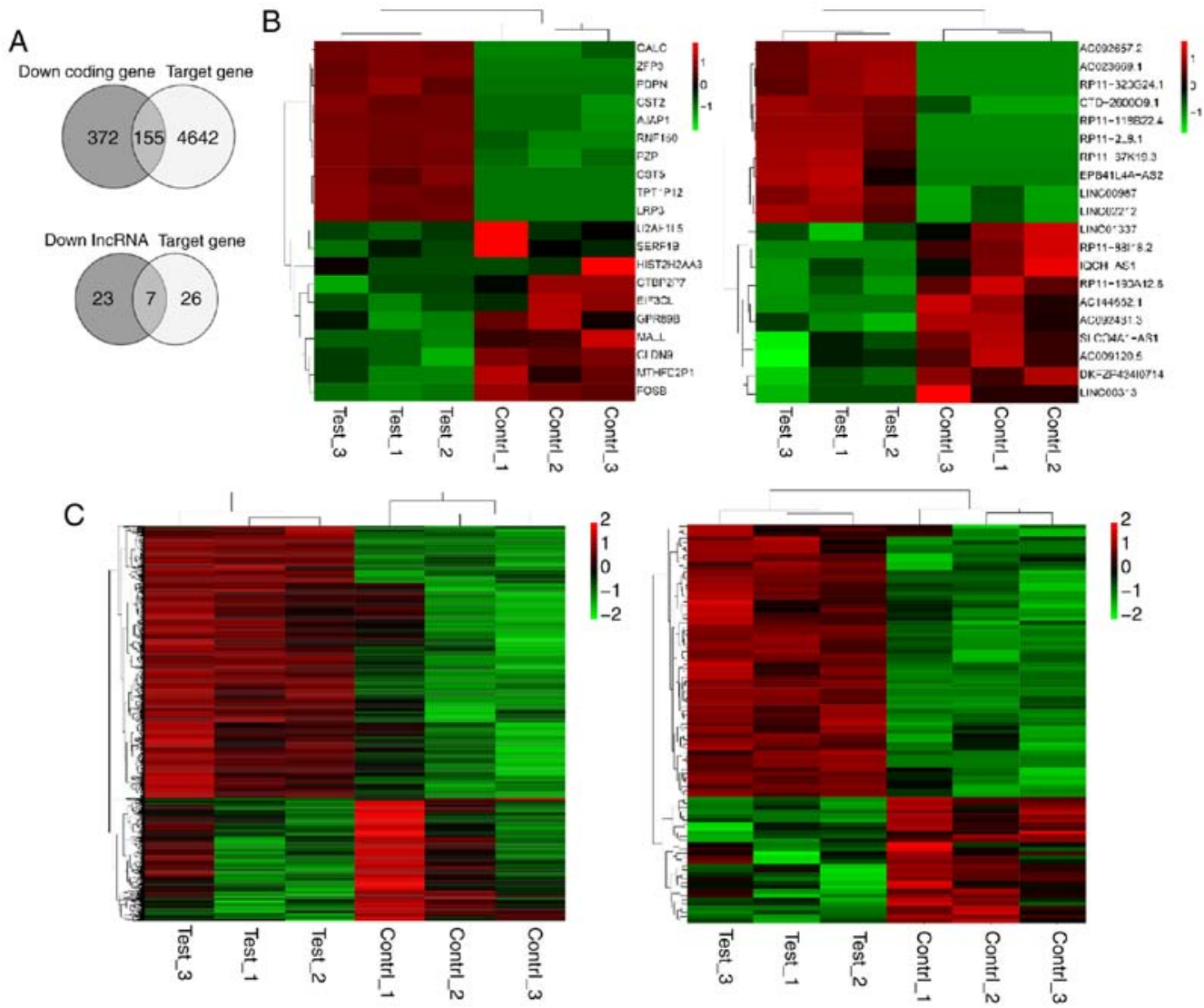

Figure 2. Analysis of DEGs. (A) VENN diagram indicating overlapping lncRNAs and genes. (B) Heat map of the top 10 DEGs; mRNA (left) and lncRNA (right). (C) Heat map of all DEGs; mRNA (left) and lncRNA (right). DEG, differentially expressed gene; lncRNA, long non-coding RNA.

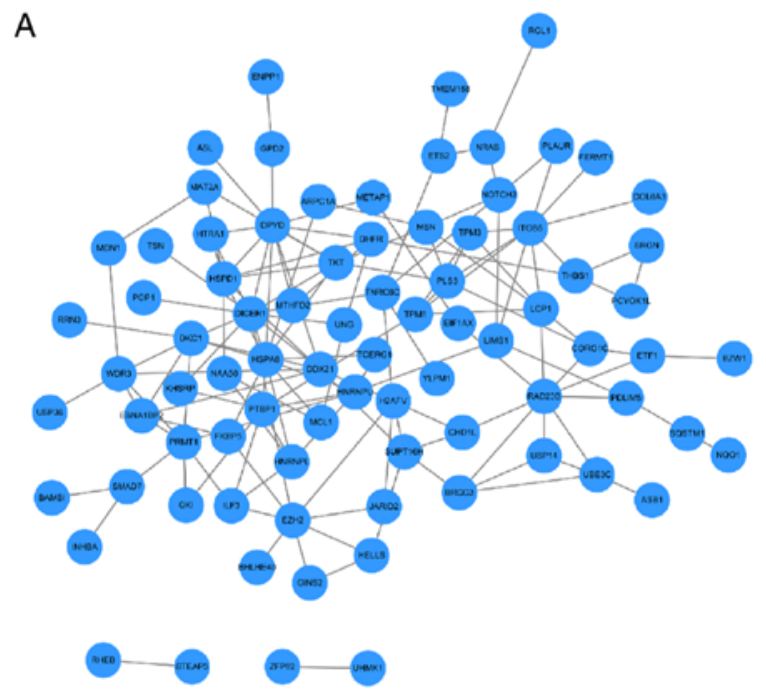

B

$\begin{array}{llll}\text { Coding gene } & \text { Degree } & \text { Coding gene } & \text { Degree } \\ \text { HSPA8 } & 12 & \text { PRMT1 } & 7 \\ \text { DICER1 } & 12 & \text { TKT } & 7 \\ \text { DPYD } & 11 & \text { HNRNPU } & 6 \\ \text { ITGB5 } & 9 & \text { H2AFV } & 6 \\ \text { RAD23B } & 9 & \text { WDR3 } & 6 \\ \text { EZH2 } & 8 & \text { PLS3 } & 6 \\ \text { HSPD1 } & 8 & \text { LCP1 } & 6 \\ \text { DDX21 } & 8 & \text { HNRNPL } & 5 \\ \text { PTBP1 } & 7 & \text { EBNA1BP2 } & 5 \\ \text { DKC1 } & 7 & \text { TPM1 } & 5\end{array}$

Figure 3. (A) Protein-protein interaction network of potential microRNA-942 target mRNAs. (B) Degree of different coding gene interactions. enrichment result. GSEA analysis revealed significant enrichment for 'vasopressin regulated water reabsorption', 'proximal tubule bicarbonate reclamation', 'fatty acid metabolism' and 'Parkinson's disease' (Fig. 5 and Table III).

Identification of prognostic factors. A total of 533 patient samples were categorized into high- and low-expression groups based on the normalized expression values of the identified lncRNAs/mRNAs. Using Kaplan-Meier analysis, the high expression levels of 31 lncRNAs and/or mRNAs were shown to result in a significant increase in patient survival rate; the most significant 22 of the 31 lncRNAs and/or mRNAs are shown in Table IV. These included the lncRNA LINC00461 and the genes spalt-like transcription factor 1 (SALL1), methionyl aminopeptidase 1 (METAP1), and DDB1 and CUL4 associated factor 11 (DCAF11) (Fig. 6).

Construction of an miR-942-related ceRNA network. Following integrated analysis of the miR-942 target genes, and Pearson's correlation analysis between miR-942 and the identified IncRNA/mRNAs, 31 potential target genes were identified for miR-942 (29 mRNAs and 2 lncRNAs). A miR-942-related ceRNA network was subsequently constructed (Fig. 7).

$R T-q P C R$ analysis of miR-942 target gene expression. The expression levels of LINC00461, miR-942, SALL1, METAP1 and $D C A F 11$ were further verified by RT-qPCR. The expression levels of miR-942 in OS-RC-2 cells were significantly 


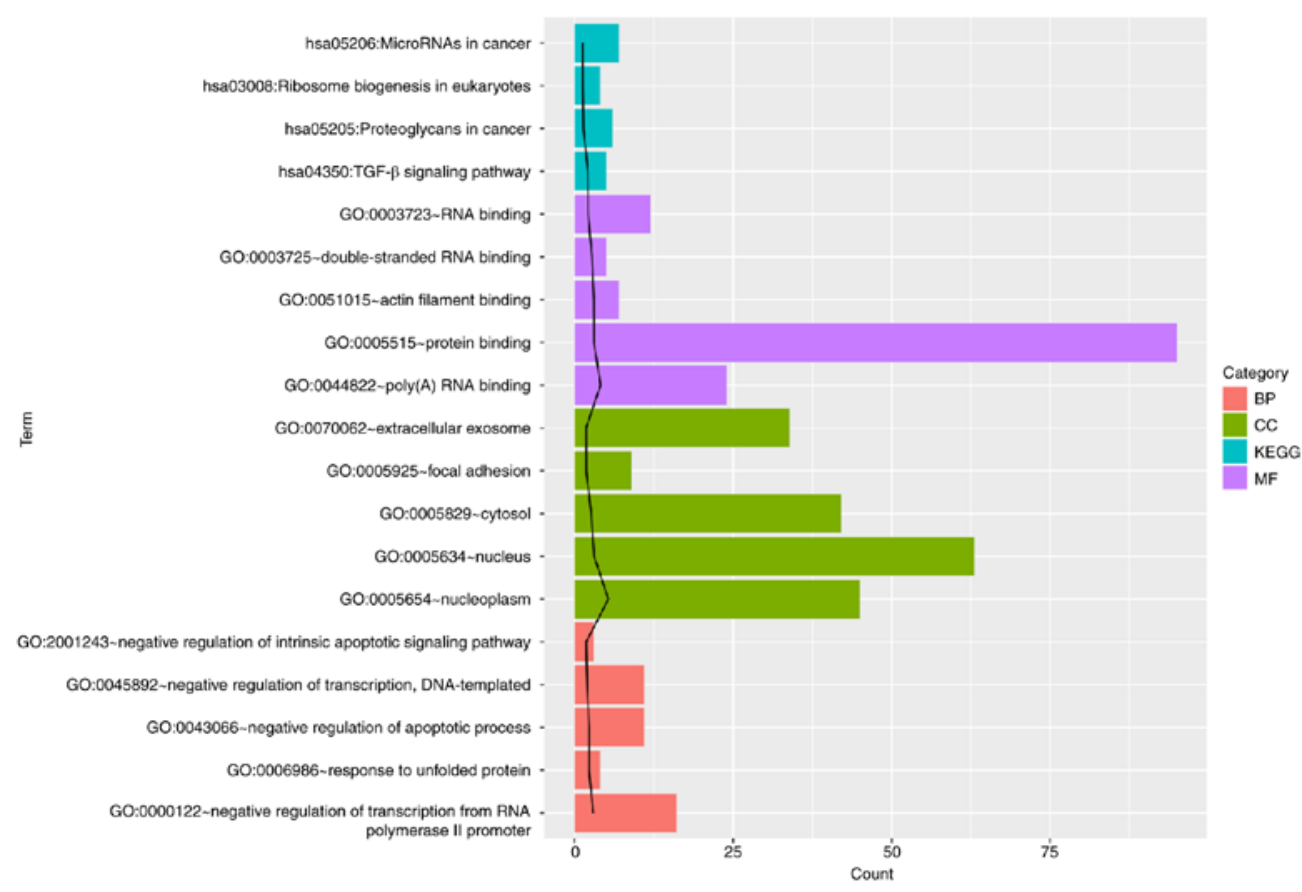

Figure 4. GO term and Kyoto Encyclopedia of Genes and Genomes pathway analysis of miRNA-942 target mRNAs. The top five GO terms or pathways are shown. The abscissa count is the number of genes enriched in each term, and the black trend line represents the value of -log 10 P-value; the larger the value, the more significant the enrichment. GO, Gene Ontology; miR, microRNA; BP, biological process; CC, cellular component; MF, molecular function.
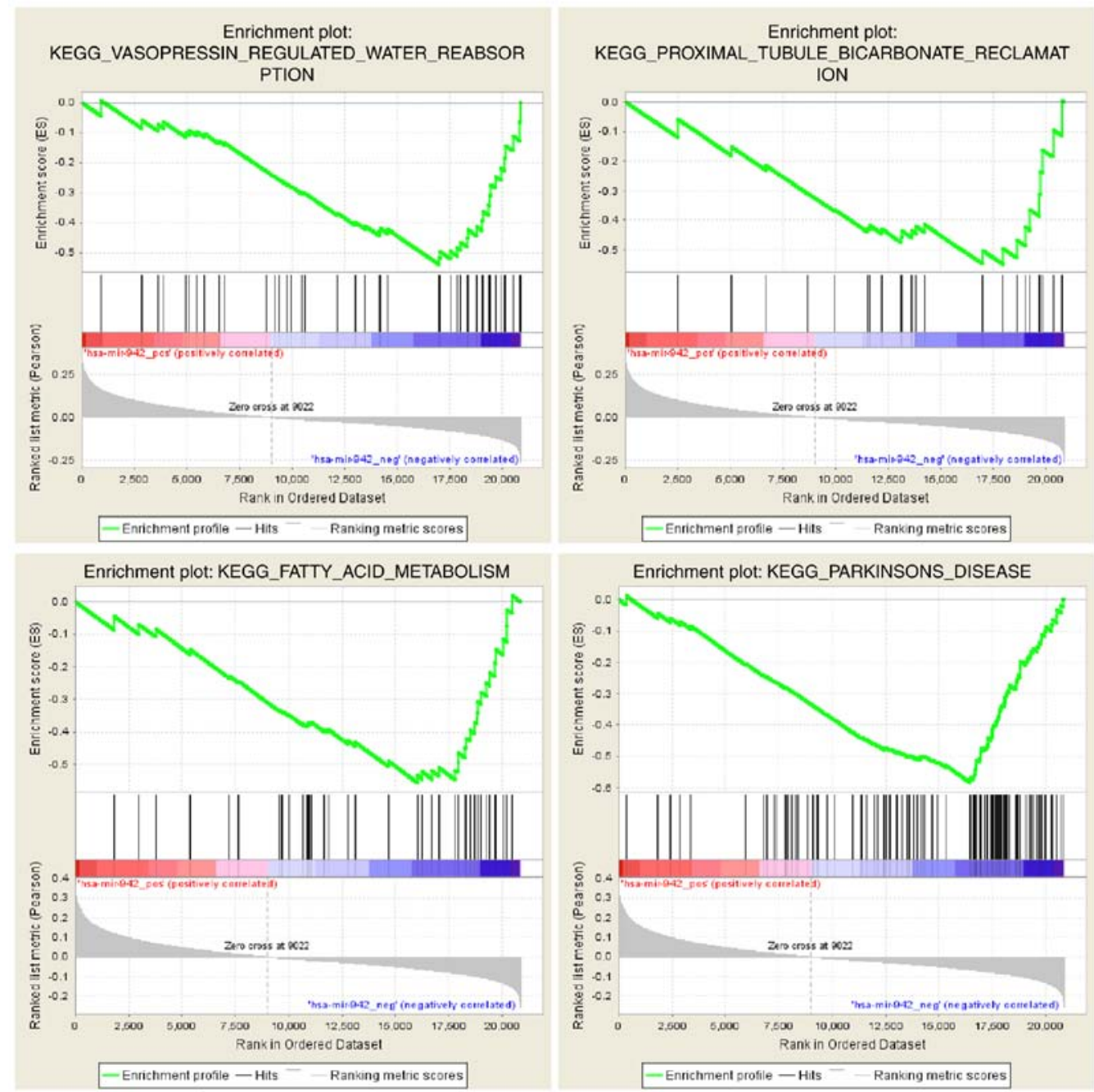

Figure 5. Gene set enrichment analysis. 
Table III. Gene set enrichment analysis of microRNA-942 and RNA-seq data from The Cancer Genome Atlas.

\begin{tabular}{lcccc}
\hline Process & Size & ES & NES & NOM p-val \\
\hline Vasopressin regulated water reabsorption & 43 & -0.53886 & -1.72647 & 0.004504505 \\
Proximal tubule bicarbonate reclamation & 23 & -0.54862 & -1.60212 & 0.040983606 \\
Fatty acid metabolism & 42 & -0.55580 & -1.54426 & 0.092702170 \\
Parkinson's disease & 112 & -0.58263 & -1.56579 & 0.098837210
\end{tabular}

Size, total genes in set; ES, enrichment score; NES, normalized enrichment score; NOM P-val, nominal P-value.

Table IV. Long non-coding RNAs or protein-coding genes that significantly effect patient survival.

\begin{tabular}{lc}
\hline Gene & P-value \\
\hline SSFA2 & $4.61 \times 10^{-11}$ \\
SALL1 & $9.32 \times 10^{-09}$ \\
BCAR3 & $1.38 \times 10^{-05}$ \\
TBC1D14 & $3.54 \times 10^{-05}$ \\
METAP1 & $3.80 \times 10^{-05}$ \\
MBLAC2 & $5.96 \times 10^{-05}$ \\
DCAF11 & $6.49 \times 10^{-05}$ \\
LINC00461 & $1.02 \times 10^{-04}$ \\
H2AFV & $5.81 \times 10^{-04}$ \\
PDIK1L & $6.82 \times 10^{-04}$ \\
LZTFL1 & $1.44 \times 10^{-03}$ \\
PTMA & $2.07 \times 10^{-03}$ \\
PLEKHB2 & $3.23 \times 10^{-03}$ \\
HMGCR & $3.63 \times 10^{-03}$ \\
TAF9B & $5.08 \times 10^{-03}$ \\
ARSD & $6.67 \times 10^{-03}$ \\
TMBIM6 & $9.49 \times 10^{-03}$ \\
DPY19L3 & $1.58 \times 10^{-02}$ \\
STARD7 & $2.11 \times 10^{-02}$ \\
SEPT2 & $2.91 \times 10^{-02}$ \\
DKFZP434I0714 & $3.00 \times 10^{-02}$ \\
ZNF552 & $4.55 \times 10^{-02}$ \\
\hline
\end{tabular}

upregulated compared with the NC mimic group (Fig. 8A; $\mathrm{P}<0.01)$. The expression level of LINC00461 was also significantly increased in the miR-942-transfected group (Fig. 8B; $\mathrm{P}<0.01)$. However, the expression levels of the miR-942 target genes SALL1, METAPI and DCAF11 were significantly downregulated in the miR-942 mimic-transfected group compared with the control-transfected group (Fig. 8C-E; $\mathrm{P}<0.01$ ). Additionally, a miR-942 inhibitor was transfected into sunitinib-resistant OS-RC-2 cells, and the expression levels of LINC00461, miR-942, SALL1, METAP1 and DCAF11 were reversed compared with the miR-942 mimic-transfected group (Fig. 8F-J; P<0.01).

MTT assay. The viability of OS-RC-2 cells was measured using an MTT assay following miR-942 transfection. As shown in Fig. 9, the viability of OS-RC-2 cells transfected with miR-942 mimics was significantly higher at $10 \mu \mathrm{mol} / 1$ sunitinib compared with that in untreated cells $(\mathrm{P}<0.01)$. However, the viability of OS-RC-2 cells was decreased following co-transfection with miR-942 mimics and LINC00641 siRNA at all concentrations of sunitinib, (compared with the cells transfected with miR-942 mimics alone) and was comparable to that of the untransfected cells. These results suggest that LINC00461 is associated with sunitinib resistance.

\section{Discussion}

Sunitinib is one of the most widely used drugs in the treatment of RCC. A Previous study has suggested that miR-942-5p, miR-133a-5p, miR-484 and miR-628-5p are upregulated in sunitinib-resistant RCC cells, and miR-942-5p was found to be the most upregulated (13). However, the exact mechanism was far from being elucidated. In the present study, miR-942 mimics were transfected into RCC cells, and MTT analysis demonstrated that cell viability was significantly increased following treatment with sunitinib, compared with that in negative control group. This result suggested that miR-942 may serve important roles in RCC cell proliferation and sunitinib resistance. In addition, RNA sequencing was performed to identify the target genes of miR-942. When comparing miR-942 mimic-treated with control-treated samples, a total of seven lncRNAs and 155 mRNAs were predicted to be target genes for miR-942. These genes were significantly associated with 'protein binding', 'TGF- $\beta$ signaling pathway', 'negative transcriptional regulation' and 'RNA binding'. Using integrated analysis of the RNA-sequencing data and that downloaded from TCGA, an miR-942-related ceRNA network was constructed and included the IncRNA LINC00461, as well as the genes SALL1, METAP1, and DCAF11, which were predicted to have a significant effect on the survival of patients with RCC.

High miR-942 expression levels in metastatic RCC cells have been demonstrated to upregulate the secretion of matrix metalloproteinase-9 and VEGF, promoting endothelial cell migration and sunitinib resistance (13). In the present study, the overexpression of miR-942 in sunitinib-sensitive OS-RC-2 cells was found to affect the expression of a number of lncRNAs and mRNAs, including lncRNA LINC00461. While few studies have reported a role for LINC00461, a recent study revealed that LINC00461 was highly expressed in glioma tissues, and is important for the proliferation and migration of glioma cells (28). lncRNAs can act as miRNA 

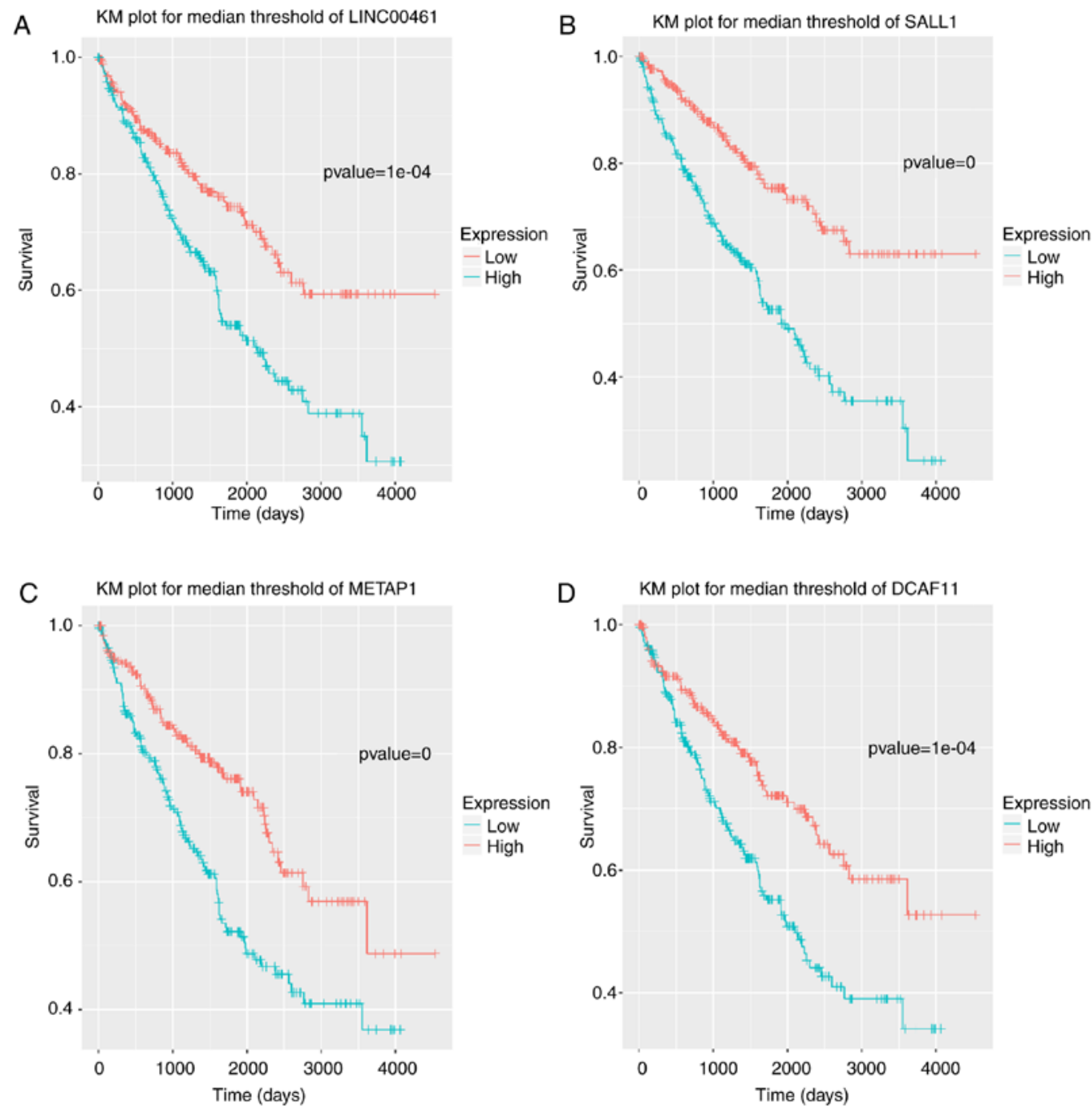

Figure 6. Kaplan-Meier survival analysis of (A) LINC00461, (B) SALL1, (C) METAP1 and (D) DCAF11 using data from The Cancer Genome Atlas. SALL1, spalt-like transcription factor 1; METAP1, methionyl aminopeptidase 1; DCAF11, DDB1 and CUL4 associated factor 1.

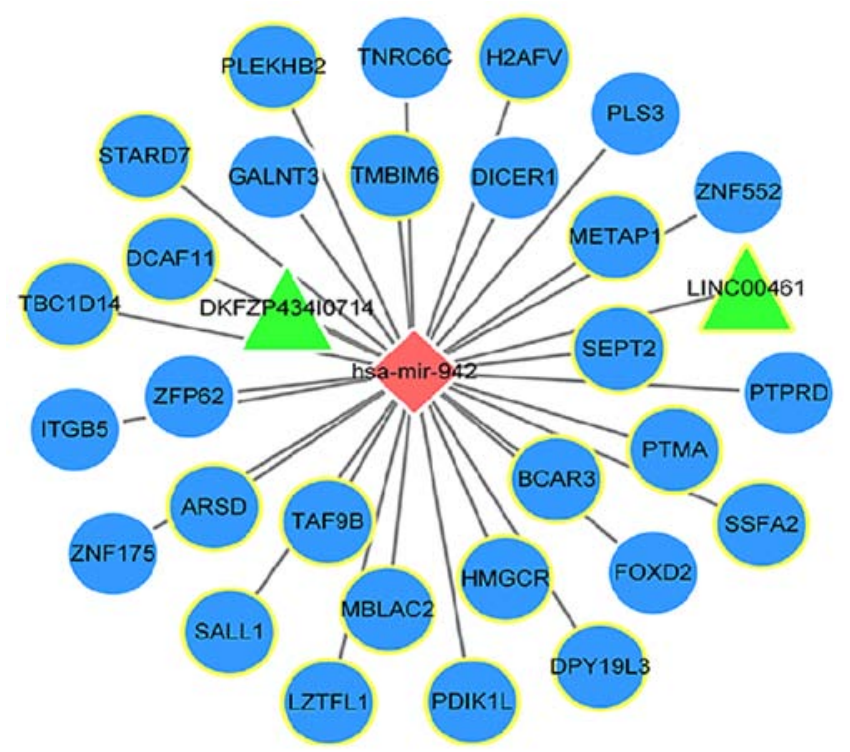

Figure 7. Construction of a miR-942-related competing endogenous RNA network. The red triangle node represents miRNA; green diamond nodes represent lncRNA; blue circular nodes represent protein-coding genes; and nodes with s yellow outer ring represent lncRNA/mRNAs that significantly affect patient survival. miR, microRNA; lncRNA, long non-coding RNA. decoys, reducing their regulatory effect on target mRNAs. In addition, studies have indicated a role for miRNA-lncRNA interactions in cancer progression (29-31). In the present study, it was speculated that LINC00461 may be a prognostic factor for RCC that may regulate miR-942. Therefore, the interaction between miR and 942-LINC00461 may serve a key role in RCC tumorigenesis, metastasis and drug resistance, which should be further verified by additional experiments.

Moreover, miR-942 was predicted to regulate the expression of the prognosis-associated factors SALL1, METAP1 and $D C A F 11$. The protein encoded by $S A L L 1$ is a zinc finger transcriptional repressor that has been shown to regulate the normal renal progenitor cell population. It is overexpressed in embryonic kidney malignancy, Wilms' tumor (WT) and progressive stem-like tumor xenografts derived from primary WT, which contributes to nephron formation and regeneration, and is indicated in WT oncogenesis (32), and METAP1 co-translationally removes the N-terminal methionine from nascent proteins (33). In addition, human METAP1 is reported to exert anti-proliferative activities in different cancer cell lines, thus may be a potent anti-cancer agent (33). A study also showed that DCAF11 mediates the degradation of the stem-loop binding protein at the end of the $\mathrm{S}$ phase, which is 

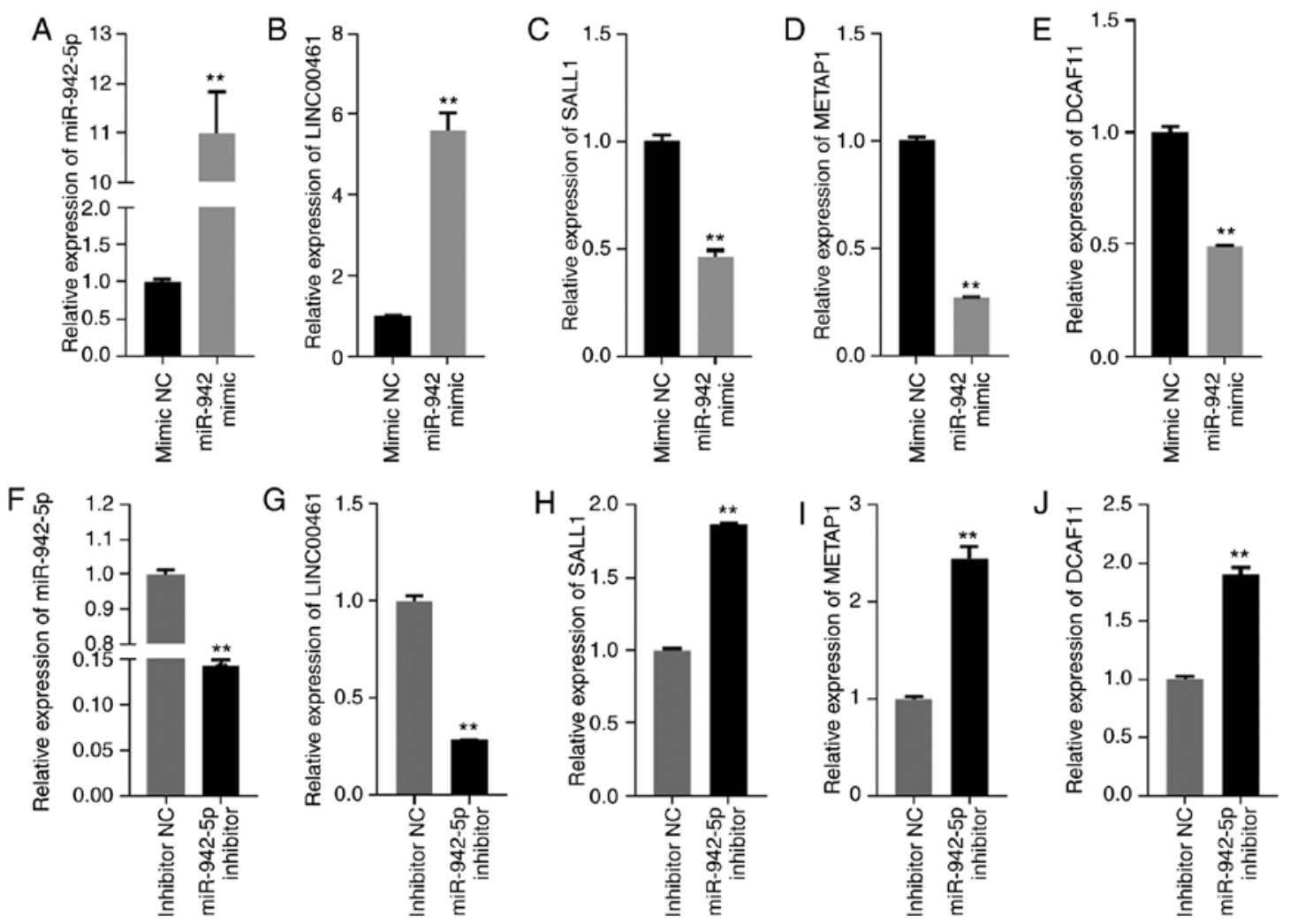

Figure 8. (A-E) Relative expression levels of miR-942, LINC00461, SALL1, METAP1 and DCAF11 in OS-RC-2 cells transfected with miR-942 mimics or the NC. (F-J) The relative expression levels of miR-942, LINC00461, SALL1, METAP1 and DCAF11 in sunitinib-resistant OS-RC-2 cells transfected with miR-942 inhibitor and the inhibitor NC. ${ }^{* * *} \mathrm{P}<0.01$. miR, microRNA; SALL1, spalt-like transcription factor 1; METAP1, methionyl aminopeptidase 1; DCAF11, DDB1 and CUL4 associated factor 1; NC, negative control.

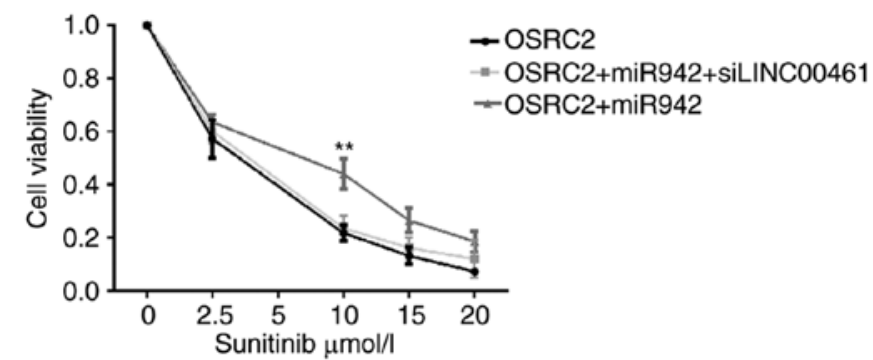

Figure 9. MTT assay. ${ }^{* *} \mathrm{P}<0.01$. OSRC2+miR942+siLINC00461 vs. OSRC2+miR942.

essential for cell viability (34). Therefore, it was speculated that miR-942 may interact with these potential target genes in the progression of RCC. Further MTT experiments demonstrated that the viability of OS-RC-2 cells was decreased following co-transfection with miR-942 mimics and LINC00641 siRNA, which was comparable with that of untransfected cells.

In conclusion, the present study indicated that miR-942 may interact with IncRNA LINC00461 and the SALL1, METAP1 and DCAF11 genes. Ongoing studies with these molecular markers may result in the generation of novel therapeutics for the prevention and treatment of RCC.

\section{Acknowledgements}

Not applicable.

\section{Funding}

The study was supported by the National Natural Science Foundation of Zhejiang Province (grant no. LY18H160002).

\section{Availability of data and materials}

All data generated or analyzed during the present study are included in this published article.

\section{Authors' contributions}

GD and $\mathrm{YiC}$ conceived and designed the study and the experiments. YiC acquired the funding. YiC, HW, WG, YL and $\mathrm{YuC}$ performed the experiments. JH and CS analyzed the data and conducted the statistical analysis. HW and $\mathrm{YuC}$ wrote the manuscript. WG and YL critically revised the manuscript. All authors read and approved the manuscript and agree to be accountable for all aspects of the research in ensuring that the accuracy or integrity of any part of the work are appropriately investigated and resolved.

\section{Ethics approval and consent to participate}

Not applicable.

\section{Patient consent for publication}

Not applicable. 


\section{Competing interests}

The authors declare that they have no competing interests.

\section{References}

1. Hsieh JJ, Purdue MP, Signoretti S, Swanton C, Albiges L, Schmidinger M, Heng DY, Larkin J and Ficarra V: Renal cell carcinoma. Nat Rev Dis Primers 3: 17009, 2017.

2. Wong MCS, Goggins WB, Yip BHK, Fung FDH, Leung C, Fang Y, Wong SYS and Ng CF: Incidence and mortality of kidney cancer: Temporal patterns and global trends in 39 countries. Sci Rep 7: 15698, 2017.

3. Walsh N, Larkin A, Kennedy S, Connolly L, Ballot J, Ooi W, Gullo G, Crown J, Clynes M and O'Driscoll L: Expression of multidrug resistance markers ABCB1 (MDR-1/P-gp) and ABCC1 (MRP-1) in renal cell carcinoma. BMC Urol 9: 6, 2009.

4. Choueiri TK, Escudier B, Powles T, Mainwaring PN, Rini BI, Donskov F, Hammers H, Hutson TE, Lee JL, Peltola K, et al: Cabozantinib versus everolimus in advanced renal-cell carcinoma. N Engl J Med 373: 1814-1823, 2015.

5. Amin A, Dudek AZ, Logan TF, Lance RS, Holzbeierlein JM, Knox JJ, Master VA, Pal SK, Miller WH Jr, Karsh LI, et al: Survival with AGS-003, an autologous dendritic cell-based immunotherapy, in combination with sunitinib in unfavorable risk patients with advanced renal cell carcinoma (RCC): Phase 2 study results. J Immunother Cancer 3: 14, 2015.

6. Joosten S, Hamming L, Soetekouw P, Aarts M, Veeck J, van Engeland $M$ and Tjan-Heijnen VC: Resistance to sunitinib in renal cell carcinoma: From molecular mechanisms to predictive markers and future perspectives. Biochim Biophys Acta 1855 $1-16,2015$.

7. Ma R, Jiang $T$ and Kang $X$ : Circulating microRNAs in cancer: Origin, function and application. J Exp Clin Cancer Res 31: 38, 2012.

8. Lim JH, Song MK, Cho Y, Kim W, Han SO and Ryu JC: Comparative analysis of microRNA and mRNA expression profiles in cells and exosomes under toluene exposure. Toxicol In Vitro 41: 92-101, 2017

9. Takahashi RU, Prieto-Vila M, Hironaka A and Ochiya T: The role of extracellular vesicle microRNAs in cancer biology. Clin Chem Lab Med 55: 648-656, 2017.

10. Goto Y, Kurozumi A, Nohata N, Kojima S, Matsushita R, Yoshino H, Ishida Y, Ichikawa T, Naya Y and Seki N: The microRNA signature of patients with sunitinib failure: Regulation of UHRF1 pathways by microRNA-101 in renal cell carcinoma. Oncotarget 7: 59070-59086, 2016.

11. Liang T, Hu XY, Li YH, Tian BQ, Li ZW and Fu Q: MicroRNA-21 regulates the proliferation, differentiation, and apoptosis of human renal cell carcinoma cells by the mTOR-STAT3 signaling pathway. Oncol Res 24: 371-380, 2016.

12. Machackova T, Mlcochova H, Stanik M, Dolezel J, Fedorko M, Pacik D, Poprach A, Svoboda M and Slaby O: MiR-429 is linked to metastasis and poor prognosis in renal cell carcinoma by affecting epithelial-mesenchymal transition. Tumor Biol 37: 14653-14658, 2016.

13. Prior C, Perez-Gracia JL, Garcia-Donas J, Rodriguez-Antona C, Guruceaga E, Esteban E, Suarez C, Castellano D, del Alba AG, Lozano MD, et al: Identification of tissue microRNAs predictive of sunitinib activity in patients with metastatic renal cell carcinoma. PLoS One 9: e86263, 2014.

14. Tomczak K, Czerwińska P and Wiznerowicz M. The Cancer Genome Atlas (TCGA): An immeasurable source of knowledge. Contemp Oncol (Pozn) 19: A68-A77, 2015.

15. Livak KJ and Schmittgen TD: Analysis of relative gene expression data using real-time quantitative PCR and the 2(-Delta Delta C(T)) method. Methods 25: 402-408, 2001.

16. Kim D, Langmead B and Salzberg SL: HISAT: A fast spliced aligner with low memory requirements. Nat Methods 12 357-360, 2015.
17. Chen Y, McCarthy D, Robinson M and Smyth GK: edgeR Differential expression analysis of digital gene expression data. User's Guide, 2014. Available online at: https://www/genomatix de/online_help/help_regionminer/edgeR pdf.

18. Benjamini $\mathrm{Y}$ and Hochberg $\mathrm{Y}$ : Controlling the false discovery rate: A practical and powerful approach to multiple testing. J Royal Stat Soc Series B (Methodological) 57: 289-300, 1995.

19. Harrow J, Frankish A, Gonzalez JM, Tapanari E, Diekhans M, Kokocinski F, Aken BL, Barrell D, Zadissa A, Searle S, et al: GENCODE: The reference human genome annotation for The ENCODE Project. Genome Res 22: 1760-1774, 2012.

20. John B, Sander C and Marks DS: Prediction of human microRNA targets. Methods Mol Biol 342: 101-113, 2006.

21. von Mering C, Huynen M, Jaeggi D, Schmidt S, Bork P and Snel B: STRING: A database of predicted functional associations between proteins. Nucleic Acids Res 31: 258-261, 2003.

22. Kohl M, Wiese S and Warscheid B: Cytoscape: Software for visualization and analysis of biological networks. Methods Mol Biol 696: 291-303, 2011

23. Huang da W, Sherman BT and Lempicki RA: Systematic and integrative analysis of large gene lists using DAVID bioinformatics resources. Nat Protoc 4: 44-57, 2009.

24. Gene Ontology Consortium. Gene ontology consortium: Going forward. Nucleic Acids Res 43: D1049-D1056, 2015.

25. Subramanian A, Tamayo P, Mootha VK, Mukherjee S, Ebert BL, Gillette MA, Paulovich A, Pomeroy SL, Golub TR, Lander ES and Mesirov JP: Gene set enrichment analysis: A knowledge-based approach for interpreting genome-wide expression profiles. Proc Natl Acad Sci USA 102: 15545-15550, 2005.

26. Therneau T: A package for survival analysis in $S$. R package version 2.37-7. 2014. URL http://cran/R-project org/package=survival. 2015.

27. Zhou M, Wang X, Shi H, Cheng L, Wang Z, Zhao H, Yang L and Sun J: Characterization of long non-coding RNA-associated ceRNA network to reveal potential prognostic lncRNA biomarkers in human ovarian cancer. Oncotarget 7: 12598-12611, 2016.

28. Yang Y, Ren M, Song C, Li D, Soomro SH, Xiong Y, Zhang H and $\mathrm{Fu} \mathrm{H}$ : LINC00461, a long non-coding RNA, is important for the proliferation and migration of glioma cells. Oncotarget 8: 84123-84139, 2017

29. Braconi C, Kogure T, Valeri N, Huang N, Nuovo G, Costinean S, Negrini M, Miotto E, Croce CM and Patel T: microRNA-29 can regulate expression of the long non-coding RNA gene MEG3 in hepatocellular cancer. Oncogene 30: 4750-4756, 2011.

30. Liang WC, Fu WM, Wong CW, Wang Y, Wang WM, Hu GX, Zhang L, Xiao LJ, Wan DC, Zhang JF and Waye MM: The lncRNA H19 promotes epithelial to mesenchymal transition by functioning as miRNA sponges in colorectal cancer. Oncotarget 6: 22513-22525, 2015.

31. Ma MZ, Chu BF, Zhang Y, Weng MZ, Qin YY, Gong W and Quan ZW: Long non-coding RNA CCAT1 promotes gallbladder cancer development via negative modulation of miRNA-218-5p. Cell Death Dis 6: e1583, 2015.

32. Metsuyanim S, Pode-Shakked N, Schmidt-Ott KM, Keshet G, Rechavi G, Blumental D and Dekel B: Accumulation of malignant renal stem cells is associated with epigenetic changes in normal renal progenitor genes. Stem Cells 26: 1808-1817, 2008.

33. Frottin F, Bienvenut WV, Bignon J, Jacquet E, Vaca Jacome AS, Van Dorsselaer A, Cianferani S, Carapito C, Meinnel T and Giglione C: MetAP1 and MetAP2 drive cell selectivity for a potent anti-cancer agent in synergy, by controlling glutathione redox state. Oncotarget 7: 63306-63323, 2016.

34. Djakbarova U, Marzluff WF and Köseoğlu MM: DDB1 and CUL4 associated factor 11 (DCAF11) mediates degradation of stem-loop binding protein at the end of $\mathrm{S}$ phase. Cell Cycle 15: 1986-1996, 2016.

This work is licensed under a Creative Commons Attribution-NonCommercial-NoDerivatives 4.0 International (CC BY-NC-ND 4.0) License. 